bioRxiv preprint doi: https://doi.org/10.1101/2019.12.11.872218; this version posted December 12, 2019. The copyright holder for this preprint (which was not certified by peer review) is the author/funder, who has granted bioRxiv a license to display the preprint in perpetuity. It is made available under aCC-BY 4.0 International license.

\title{
Measurements of 3-D morphology of individual red blood cells upon localized photothermal effects using gold nanorods
}

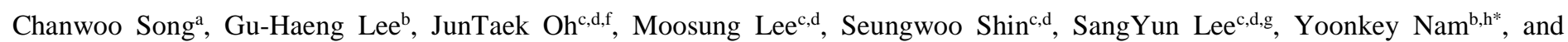

YongKeun Park ${ }^{\mathrm{c}, \mathrm{d}, \mathrm{e}^{*}}$

a Department of Materials Science and Engineering, Korea Advanced Institute of Science and Technology (KAIST), Daejeon,

Republic of Korea;

b Department of Bio and Brain Engineering, KAIST, Daejeon, Republic of Korea;

c Department of Physics, KAIST, Daejeon, Republic of Korea;

${ }^{\mathrm{d}}$ KAIST Institute for Health Science and Technology, Daejeon, Republic of Korea;

e Tomocube Inc., Daejeon, Republic of Korea

${ }^{\mathrm{f}}$ Current affiliation: Samsung Mechatronics, Suwon, Republic of Korea

g Current affiliation: Samsung Electronics, Suwon, Republic of Korea

h E-mail: ynam@kaist.ac.kr

*yk.park@kaist.ac.kr

(a)

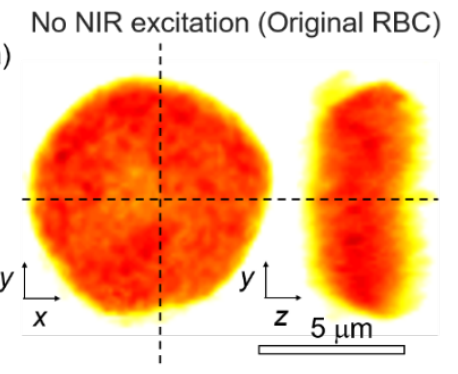

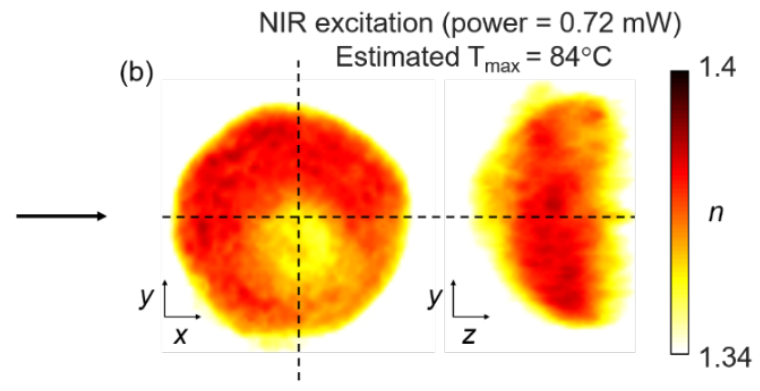

(e) (c)

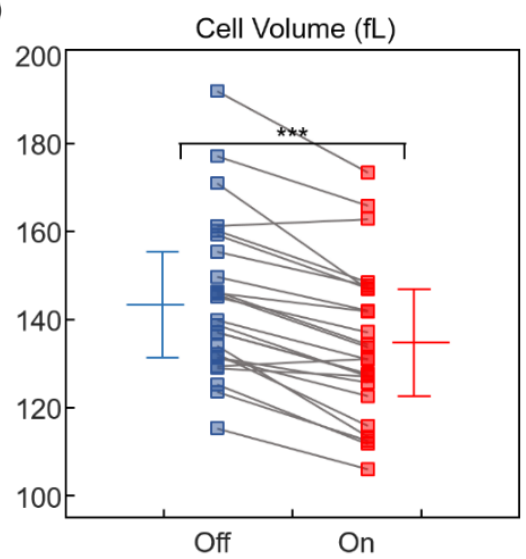

(d)

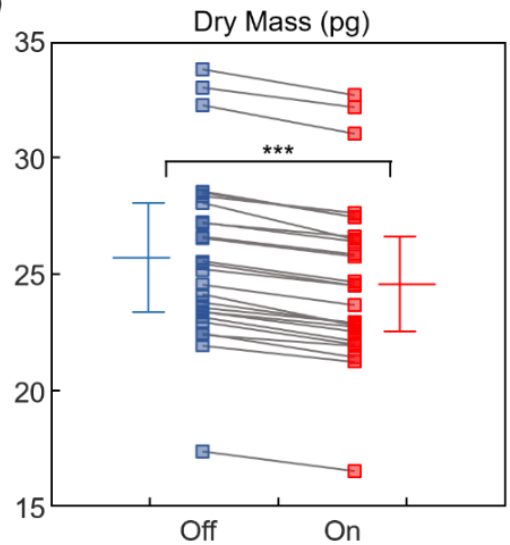

$\mathrm{Hb}$ Concentration (g/dL)

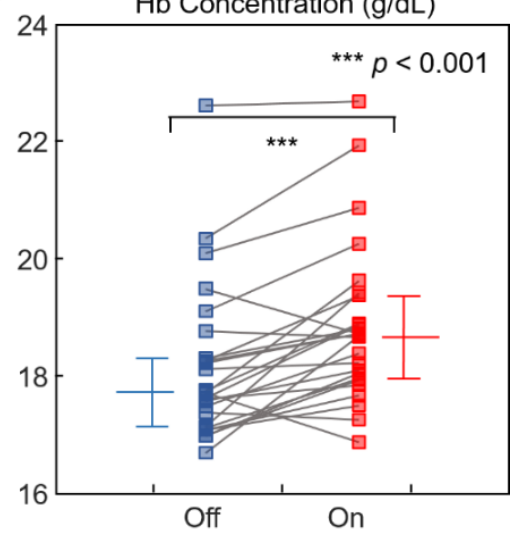




\section{ABSTRACT}

We investigated the in-situ photothermal response of human red blood cells (RBCs) by combining photothermal heat generation and 3D quantitative phase imaging techniques. Gold-nanorod-coated substrates were excited using near-infrared light to generate local heat to RBCs, and response was measured by imaging 3-D refractive index tomograms of cells under various near infrared (NIR) excitation conditions. On photothermal treatment, cell morphology changed from discoid to crescent shapes, cell volume and dry mass decreased, and hemoglobin concentration increased. We also investigated the irreversible deformation of RBCs when multiple intense excitation shocks are applied. These results provide a new understanding of thermodynamic aspects of cell biology and hematology.

\section{INTRODUCTION}

Alterations in the biophysical properties of red blood cells (RBCs) are directly related to various diseases such as sickle cell disease ${ }^{1,2}$, malaria $^{3-6}$, and diabetes ${ }^{7,8}$, and can be potential indicators for diagnosing various diseases ${ }^{9,10}$. The thermal response of RBCs can be an effective indicator for the pathophysiology of various diseases. Even though it is well known that the pathophysiology of many RBC related diseases is temperature related ${ }^{3}$, most previous studies have been limited to a small temperature range (between room temperature and body or fever temperature) ${ }^{3}$. Furthermore, a systematic investigation of the cellular response to local heating of individual cells, particularly using three-dimensional imaging, has not been explored. This is unfortunate because it could provide valuable information to connect the pathophysiology of diseases to thermodynamics, and unveil complex, non-equilibrium thermodynamic behavior ${ }^{11-15}$.

The study of the thermal response of RBCs at an individual cell level has been hindered by technical limitations of heat treatment and imaging methods. First, local heat treatment of biological cells is inaccessible using a conventional heating chamber. Most previous thermodynamic experiments on RBCs have relied upon ensemble averaging of multiple cells, such as a case using a microfluidic pipette ${ }^{16}$. Furthermore, rapid three-dimensional (3-D) time-lapse imaging of RBCs is required to study cellular response upon local heating. However, imaging the 3-D dynamics of RBCs over a long time at a high frame rate is challenging for either conventional bright-field or fluorescence-based microscopy. Owing to these limitations, conventional imaging approaches have provided limited access to the morphological and biochemical properties of RBCs, such as lateral shapes and out-of-membrane fluctuations ${ }^{17-19}$.

To directly investigate the in-situ thermal response of individual RBCs, we present a novel combination of two optical approaches: photothermal heat generation using gold nanorods (GNRs) and 3-D rapid quantitative imaging using a 3-D quantitative phase imaging technique (QPI). The GNR-coated coverslips, on which RBCs are located, are locally excited by illuminating a tightly focused optical spot using an $808 \mathrm{~nm}$ wavelength near-infrared (NIR) laser. The NIR excitation beam locally generates heating of GNRs via surface plasmonic resonance ${ }^{20,21}$. Due to conduction, the temperature of the RBCs rapidly increases and induces a thermal response from 
individual RBCs. Then, in order to measure the 3-D response of individual RBCs, we employ optical diffraction tomography (ODT), one of several 3-D QPI techniques ${ }^{22,23}$. QPI is a label-free imaging technique using the refractive index (RI) for intrinsic and quantitative imaging contrast ${ }^{22,24}$. By utilizing interferometric or non-interferometric imaging systems, QPI has been applied to various applications, including hematology ${ }^{25-28}$, malaria infection ${ }^{3,29,30}$, neuroscience ${ }^{31,32}$, cell biology ${ }^{33-35},{ }^{36}$ reproductive biology ${ }^{37}$, diabetes ${ }^{7}$, and biotechnology ${ }^{38}$, due to its label-free, live, and rapid imaging capability.

Here, by measuring the 3-D RI distribution of an RBC being photothermally excited, various biophysical parameters of the target cell are systematically investigated. We observed and quantitatively analyzed the morphological and biophysical changes of individual RBCs under local heat exposure in situ. In this report, we suggest that the integration of the ODT technique and photothermal heat generation serves as a novel method for extensive study of various single-cell thermodynamics.

\section{METHODS AND MATERIALS}

\section{Experimental Setup}

The experimental setup and the analysis procedure are illustrated in Fig. 1. RBCs dispersed in Alsever's solution were sandwiched between two No.1-thickness coverslips (Fig. 1(a)). The bottom coverslip was coated with cylindrically symmetric ellipsoidal GNRs, whose diameters were $14 \mathrm{~nm}$ and $50 \mathrm{~nm}$ on the minor and major axes, respectively (Fig. 1(b)). The GNRs were designed and optimized to generate heat diffusion under NIR light at $808 \mathrm{~nm}$ and effectively raise the temperature of a sample due to its high absorption and heat conversion efficiency ${ }^{39}$ (Fig. 1(c)).

In order to simultaneously increase the local temperature and probe the 3-D response of the heated RBC, we used an ODT system (Fig. 1(d)) combined with an NIR laser for photothermal excitation and a visible laser for imaging ${ }^{39}$. To raise the temperature of individual RBCs, an NIR laser diode ( $\lambda=808$ nm, M9-808-015, Thorlabs Inc., USA) was used to excite GNRs. The NIR laser beam was focused at the center of a target RBC, with a beam diameter of $8.5 \mu \mathrm{m}$. The temperature increase was calibrated using data obtained with the GNR-coated coverslip in the absence of cells ${ }^{40}$. We assumed that the temperature of target RBCs increases immediately upon NIR illumination, based on the nature of the rapid thermal equilibrium of a micro-system ${ }^{41,42}$.

To measure 3-D images of individual RBCs upon thermal treatment, an ODT system equipped with a DMD was utilized. A He-Ne laser ( $\lambda=633 \mathrm{~nm}, 15 \mathrm{~mW}$, Thorlabs Inc., USA) was split into a reference beam and a sample beam using a $2 \times 2$ single-mode optical fiber coupler. To control illumination angles of the sample beam, a DMD (DLP LightCrafter 6500, Texas Instruments Inc., USA) was located at the conjugate plane to a sample ${ }^{43}$. Time-multiplexed hologram patterns were projected onto the DMD, to control the illumination angle of the beam impinging onto a sample, with high speed $(100 \mathrm{~Hz})$ and accuracy ${ }^{41,42}$. The modulated illumination beam was projected onto a sample using a tube lens $(\mathrm{TL} 1, f=250 \mathrm{~mm})$ and a water-immersion condenser lens (CL, NA = 1.1 LUMPLN, 60×, 
Olympus Inc., Japan). To collect the diffracted beam from the sample, the water-immersion objective lens (OL, NA = 1.2 UPLSAPO, $60 \times$, Olympus Inc., Japan) and a tube lens (TL2, $f=180 \mathrm{~mm}$ ) were used. The sample beam was then imaged on to a camera plane, where it interfered with a reference beam with a slight tilt, resulting in the formation of a spatially modulated interference pattern. The interference patterns were recorded using a CMOS sensor (FL3-U3-13Y3M-C, FLIR Systems Inc., USA). To avoid the detection of the NIR excitation laser beam at the camera, a short pass filter (edge wavelength $=750 \mathrm{~nm}$, Thorlabs Inc., USA) was placed in front of the camera.

From the recorded interference patterns of a sample illuminated at various incident angles, a 3-D RI tomogram was reconstructed using ODT principles (Fig. 1(e) ${ }^{44}$. First, optical field images, consisting of both the amplitude and phase images, were retrieved from the interference patterns using a phase retrieval algorithm ${ }^{45,46}$. Then, retrieved optical field images were mapped into a 3-D Fourier space, based on the Fourier diffraction theorem and the Rytov approximation ${ }^{23,44}$. Due to the limited numerical apertures of both the condenser and objective lenses, side scattering information was not collected, resulting in a missing cone problem. To remedy the missing cone problem, the Gerchberg-Papoulis (GP) algorithm based on a non-negativity constraint was applied ${ }^{47,48}$. The iteration number was set to 100, and it was assumed that the sample RI is higher than the medium RI. The theoretical lateral and axial resolutions are $110 \mathrm{~nm}$ and $360 \mathrm{~nm}$, respectively ${ }^{49}$. The details of ODT with the Matlab reconstruction code and the calibration of local heating can be found elsewhere ${ }^{40,50-52}$.

\section{Synthesis of gold nanorods}

A seed-mediated method was utilized to synthesize GNRs ${ }^{53}$. At room temperature, $2.5 \mathrm{ml}$ of $0.2 \mathrm{M}$ cetyltrimethylammonium bromide (CTAB, Sigma), $2.5 \mathrm{ml}$ of $0.5 \mathrm{mM} \mathrm{HAuCl}_{4}$ (Sigma), and $300 \mu \mathrm{L}$ of ice-cold $0.01 \mathrm{M} \mathrm{NaBH}_{4}$ (Sigma) were mixed in an ultrasonication bath and used as a seed solution. After aging the seed solution for $2 \mathrm{~h}$, seeds were grown to a rod shape in growth solution for 30 min at room temperature. The growth solution was a mixture of $5 \mathrm{ml} 0.2 \mathrm{M} \mathrm{CTAB}, 5 \mathrm{ml}$ of $1 \mathrm{mM} \mathrm{HAuCl}, 250 \mu \mathrm{L}$ of $4 \mathrm{mM} \mathrm{AgNO} 3\left(\mathrm{Sigma}_{4}\right.$, $70 \mu \mathrm{L}$ of $78.84 \mathrm{mM}$ ascorbic acid (Sigma) and $12 \mu \mathrm{L}$ of seed solution. The GNRs were then concentrated by centrifugation at 10,000 RPM (10,200 RCF) and resuspended in ultrapure water in order to remove the surfactant. The GNRs were coated with polyethylene glycol (mPEG-SH, MW 5000, Nanocs) with a ratio of 3 optical density (O.D.) of GNR to $3 \mathrm{mg} / \mathrm{ml}$ of PEG in water solution for $12 \mathrm{~h}$ at room temperature. Using a dialysis kit (Thermo Scientific), excess PEG was removed for two days. We measured the zeta potential of PEG-coated GNR by Zetasizer Nano ZS (Malvern).

To ensure a clean surface, glass substrates were cleaned with acetone, isopropyl alcohol, and deionized water for 5 min each with ultrasonication. Layer-by-layer coating was performed on each substrate to create a positively charged surface. For the substrate treatment, we prepared $10 \mathrm{mg} / \mathrm{ml}$ of poly(sodium 4-styrenesulfonate) (PSS, MW 70,000, Aldrich) and poly(allylamine hydrochloride) (PAH, MW 17,500, Aldrich) in $10 \mathrm{mM} \mathrm{NaCl}$ solution. Then, the glass substrates were treated with PSS and PAH solution for 3 cycles 
of 5 min each and finalized with PAH. A 1 O.D. GNR solution $\left(0.2 \mathrm{ml} / \mathrm{cm}^{2}\right)$ with a zeta potential of $-37.5 \mathrm{mV}$ was dispersed on the substrates for $12 \mathrm{~h}$. Slight mismatch of absorption peak was tuned by additional layer-by-layer coating on GNR coated substrates. The average length and diameter of synthesized GNRs are $50 \mathrm{~nm}$ and $14 \mathrm{~nm}$, respectively (aspect ratio of 3.60).

We measured the absorbance spectrum and the absorbance value at $808 \mathrm{~nm}$ by vis-NIR spectroscopy (Ocean Optics). The absorption spectrum of the GNRs was observed to have a maximum peak at around $808 \mathrm{~nm}$ (Fig. 1(c)). Using absorbance microscopy, the optical properties of the sample were also measured. The average absorbance of the samples upon excitation was shown to have an O.D. of 0.127 O.D. and an absorbance ratio of $25.4 \%{ }^{40}$.

\section{Preparation of red blood cell samples}

Blood was extracted using a lancet. A total of $5 \mu \mathrm{L}$ of blood was collected from healthy volunteers using a pipette, and then diluted in $1 \mathrm{~mL}$ of Alsever's solution (A3551, Sigma-Aldrich, Missouri, United States). For each measurement, $50 \mu \mathrm{L}$ of the diluted solution was dropped onto a GNR-coated coverslip and covered with another bare coverslip ( $24 \times 40 \mathrm{~mm}$, C024501, Matsunami, Ltd, Japan). The sample was loaded on a stage of the optical setup and measured. All the experimental protocols were approved by the institutional review board of KAIST (IRB project: KH2015-37).

\section{Morphology analysis of individual RBCs}

The morphological and biophysical parameters of individual RBCs were retrieved from measured 3-D RI tomograms. Because the RI contrast of RBCs provides their hemoglobin concentration distribution ${ }^{54}$, ODT enables label-free quantification of RBC parameters in a non-invasive manner ${ }^{55,56}$. The following cell parameters were retrieved: cell volume, hemoglobin (Hb) concentration, and cellular dry mass.

The cell volume was calculated from the number of voxels with an RI value higher than a threshold value of 1.350 . The Hb concentration of each cell, [Hb], was calculated from the measured RI values of the RBC cytoplasm. Due to a linear relationship between the mean RI value of non-aqueous cytoplasmic contents and its mean concentration ${ }^{57,58}$, the Hb concentration of an RBC can be calculated from the mean RI contrast $\langle\Delta n\rangle$ :

$$
\langle\Delta n\rangle=\langle n(x, y, z)\rangle-n_{m}=\alpha[\mathrm{Hb}]
$$

where $n_{m}$ is the RI of a medium, $\alpha$ is the refraction increment, a coefficient between the relationship between the mean RI of RBC cytoplasm and $\mathrm{Hb}$ concentration. In this work we used a value of $0.18 \mathrm{~mL} / \mathrm{g}$ for $\alpha^{54,59}$. The dry mass of individual RBCs, the mass of 
non-aqueous cytoplasmic contents, can also be obtained from integration of the Hb concentration over a cytoplasmic volume:

$$
\text { dry mass }=\int[\mathrm{Hb}] d V_{\text {cell }}=\int \frac{\langle\Delta n\rangle}{\alpha} d V_{\text {cell }}=\sum_{\text {cell }} \frac{\Delta \phi(x, y)}{2 \pi \alpha} \Delta x \Delta y \text {, }
$$

where $\Delta \phi(x, y)$ is a measured phase delay in a given coordinate and $\Delta x \Delta y$ is the area in the field of view corresponding to the pixel area of the camera.

\section{Statistical Analysis}

For a statistical comparison of the mean RBC parameters of the original and heat-treated RBC groups, a Wilcoxon rank-sum test was utilized. In experiments based on individual cell tracking, the paired Wilcoxon rank-sum test was employed to address continuous alterations in the measured parameters of individual RBCs under the heat treatment process. Throughout the manuscript, all the retrieved RBC parameters are given in the form mean \pm std, and a $p$-value of less than 0.05 was regarded as statistically significant.

\section{Results}

\section{Effects of localized heating on RBCs}

To demonstrate the effects of localized heating on RBCs, a 3-D RI tomogram of an individual RBC was measured before and two seconds after NIR excitation (Fig. 2). Laser was continuously irradiated during image acquisition, which requires 0.3 s. Representative images before and after NIR excitation are shown in Figs. 2(a-b). Before excitation, cells exhibit a characteristic biconcave shape (Fig. 2(a)). After NIR excitation (0.72 mW), a significant morphological alteration causes the overall shape to be crescent.

For a clearer comparison, these morphological and biochemical RBC responses were quantified and statistically analyzed (Figs. 2(ce)). In particular, we retrieved the statistics of volume, dry mass, and Hb concentration of 33 RBCs from their measured 3-D RI tomograms. The mean volume of RBCs significantly decreased from $142.41 \pm 20.53 \mathrm{fL}$ at room temperature $\left(25{ }^{\circ} \mathrm{C}\right)$ to $132.35 \pm 19.81$ fL after the excitation from room temperature ( $p<0.001$ ) (Fig. 2(c)). The total dry mass also decreased significantly from $25.73 \pm 3.67$ pg to $24.84 \pm 3.61 \mathrm{pg}$ after the excitation $(p<0.001$ ) (Fig. 2(d)). These data indicate that the localized photothermal heat generation induced the efflux of internal RBC components. To determine whether the major effused component is water or hemoglobin, we compared the statistics of the mean Hb concentration of RBCs before and after NIR excitation (Fig. 2(e)). The retrieved mean Hb concentrations of RBCs at RT and after localized photothermal treatment are $18.12 \pm 1.31 \mathrm{~g} / \mathrm{dL}$ and $18.83 \pm 1.35 \mathrm{~g} / \mathrm{dL}$, respectively, indicating a significant difference $(p<0.001)$. From these data, we confirmed that about $70 \%$ of volume decrease is contributed by water. 


\section{Deviation in RBC parameters is directly related to power of excitation beam for localized heat treatment}

To determine whether there is a significant correlation between the amount of photothermally generated heat and the RBC physiological changes, we examined the morphological and biochemical parameters of RBCs as a function of NIR illumination power (Fig. 3). We controlled the NIR laser power from 0.48 to $0.84 \mathrm{~mW}$ with an interval of $0.12 \mathrm{~mW}$, and quantified the relative parameter changes before and after the photothermal treatments. Same imaging procedure with what mentioned at section 3.1 was adopted in this experiment.

As shown in Fig. 3(a), we found a significant negative correlation between NIR laser power and volume. The volume decrease ratio per power was $-21.78 / \mathrm{mW}$ below $0.84 \mathrm{~mW}$. In contrast, the dry mass change with power level was inconsistent (Fig. 3(b)). Based on these statistics, the power dependence of the hemoglobin concentration was rationally fitted [Fig. 3(c)]. As expected, the fitted curve showed significant positive, rational relationship $\left(\mathrm{R}^{2}>0.99\right)$. Overall, these trends agree well with the single-cell statistics in Fig. 2 , and indicate that volume decrease and Hb concentration increase are significant features of RBCs subjected to heat treatment.

\section{Reversibility of the RBC alteration due to localized heat treatment}

We further examined the reversibility of the photothermal response of individual RBCs (Fig. 4). We illuminated a 2.3-second pulse train NIR laser beam with a time interval of $4.6 \mathrm{~s}$, and analyzed the response of individual RBCs (Fig. 4(a)). Same imaging procedure from section 3.1 was adopted for each steps of NIR exposure.

Under low NIR power $\left(0.48 \mathrm{~mW}\right.$, estimated $\left.\mathrm{T}_{\max }=72^{\circ} \mathrm{C}\right)$, we observed that all the RBCs exhibited reversible volume decreases after 5 cycles of heat treatment (Fig. 4(b)). Decreased volume of the RBCs due to heat addition was recovered instantaneously when the excitation laser was turned off (Fig. 4(b), Supplementary Video 1). However, upon high power NIR illumination (0.84 mW, estimated $\mathrm{T}_{\max }=90^{\circ} \mathrm{C}$ ), an irreversible recovery was observed after multiple heat exposure (Fig. 4(c), Supplementary Video 2). Notably, we observed that several RBCs burst when exposed to the $0.84 \mathrm{~mW}$ NIR beam (Supplementary Video 3).

We studied the heat-dependent irreversibility of RBCs by comparing the relative parameter ratios after the first and fifth exposures (Figs. 4(d-f)). The relative volume ratio statistics indicate dramatic irreversible volume decreases after five times of NIR excitation (Fig. 4(d)). This may be related to the fact that the estimated maximum temperature exceeded the typical range of protein denaturation temperature, around $40^{\circ} \mathrm{C}$. In contrast, the dry mass ratio remained almost constant except when RBCs burst at high heat levels (Fig. 4(e)). As a result, the irreversible concentration increase was more dramatic with stronger NIR laser power (Fig. 4(f)). Overall, our results suggest that irreversible changes in RBCs due to heat treatment are correlated with volumetric decrease and concentration increase.

\section{Discussions and Conclusion}


Here we presented in situ observations of morphological and biochemical alterations of RBCs subjected to localized photothermal treatment. Localized photothermal treatment was applied to individual RBCs by using NIR excitation of GNRs. The corresponding alterations of individual RBCs were precisely and quantitatively measured in 3-D using ODT. Our study revealed significant morphological changes in RBCs after localized photothermal treatments, including volume decrease, dry mass loss, and concentration increase. We also investigated the reversibility of the RBC physiological changes driven by photothermal excitation. We observed three key quantitative parameter changes depending upon local temperature increment: decreasing volume, decreasing dry mass, and increasing protein concentration. Although an overall understanding of thermally altering RBC properties remains a great challenge, our approach and findings provide an important step to address biophysical models for RBC thermodynamics. These methods can also be utilized in the context of cellular physiology.

The physiological origin of these changes is still unclear, so we anticipate that the following hypotheses can be tested in future studies. First, the volume decrease of RBCs under heat treatment may be explained in association with larger water permeability across the RBC membrane at higher temperature ${ }^{60,61}$. Second, the dry mass loss of RBCs at temperatures higher than $40^{\circ} \mathrm{C}$ is very likely a result of protein denaturation in the RBCs. In order to validate these hypotheses, biochemical analysis tools should be used for further exploration.

Although we focused on the heat response of RBCs, the proposed method is generally applicable to studying the physiology of other types of cells. Because GNRs have been conventionally applied in photothermal therapy, it would be intriguing to investigate singlecell studies related to cancer treatment ${ }^{20}$, neuron signal modulation ${ }^{56,62,63}$, and neuron growth ${ }^{64}$. Furthermore, the responses of individual RBCs at high temperatures can also be utilized for in vivo photothermal therapy ${ }^{65}$. Also, the throughput of the proposed system can be extended by combining a wide-field imaging platform. In such systems, cell-cell interactions under local heat treatment could also be investigated. Overall, the proposed method opens new horizons for understanding the thermodynamic physiology of biological systems.

\section{Acknowledgments}

This work was supported by KAIST, BK21+ program, Tomocube, and National Research Foundation of Korea (2017M3C1A3013923, 2015R1A3A2066550, 2018K000396). The authors thank Dr. Weisun Park for helpful discussions.

\section{COMPETING FINANCIAL INTERESTS}

Prof. Park and Mr. Moosung Lee have financial interests in Tomocube Inc., a company that commercializes optical diffraction tomography and quantitative phase imaging instruments and is one of the sponsors of the work.

\section{References}


Shaked, N. T., Satterwhite, L. L., Truskey, G. A., Wax, A. P. \& Telen, M. J. Quantitative microscopy and nanoscopy of sickle red blood cells performed by wide field digital interferometry. Journal of biomedical optics 16, 030506 (2011).

Byun, H. et al. Optical measurement of biomechanical properties of individual erythrocytes from a sickle cell patient. Acta biomaterialia 8, 4130-4138 (2012).

Park, Y. et al. Refractive index maps and membrane dynamics of human red blood cells parasitized by Plasmodium falciparum. Proceedings of the National Academy of Sciences 105, 13730-13735 (2008).

Park, Y. et al. Static and dynamic light scattering of healthy and malaria-parasite invaded red blood cells. Journal of biomedical optics 15, 020506 (2010). (2012).

Lee, S. et al. Refractive index tomograms and dynamic membrane fluctuations of red blood cells from patients with diabetes mellitus. Scientific reports 7, 1039 (2017). Diez-Silva, M., Dao, M., Han, J., Lim, C.-T. \& Suresh, S. Shape and biomechanical characteristics of human red blood cells in health and disease. MRS bulletin 35, 382-388 (2010).

Park, H. et al. Characterizations of individual mouse red blood cells parasitized by Babesia microti using 3-D holographic microscopy. Scientific reports 5, 10827 (2015).

Williamson, J. R., Shanahan, M. \& Hochmuth, R. The influence of temperature on red cell deformability. Blood 46, 611-624 (1975).

Hanss, M. \& Koutsouris, D. Thermal transitions of red blood cell deformability. Correlation with membrane rheological properties. Biochimica et Biophysica Acta (BBA)-Biomembranes 769, 461-470 (1984). Hochmuth, R. \& Waugh, R. Erythrocyte membrane elasticity and viscosity. Annual review of physiology 49, 209-219 (1987). Park, Y. et al. Measurement of the nonlinear elasticity of red blood cell membranes. Physical Review E 83, 051925 (2011). Turlier, H. et al. Equilibrium physics breakdown reveals the active nature of red blood cell flickering. Nature Physics 12, 513 (2016). $J$ 29, 177-182 (1980). Tuchin, V. V. \& Tuchin, V. Tissue optics: light scattering methods and instruments for medical diagnosis. (2007). 2010).

Evans, J., Gratzer, W., Mohandas, N., Parker, K. \& Sleep, J. Fluctuations of the red blood cell membrane: relation to mechanical properties and lack of ATP dependence. Biophys J 94, 4134-4144 (2008).

Huang, X. \& El-Sayed, M. A. Gold nanoparticles: optical properties and implementations in cancer diagnosis and photothermal therapy. Journal of advanced research 1, 13-28 (2010). 10, 25 (2015). Park, Y., Depeursinge, C. \& Popescu, G. Quantitative phase imaging in biomedicine. Nature Photonics 12, 578-589, doi:10.1038/s41566-018-0253-x (2018).

Kim, K. et al. Optical diffraction tomography techniques for the study of cell pathophysiology. Journal of Biomedical Photonics \& Engineering 2 (2016).

Kim, Y. et al. Profiling individual human red blood cells using common-path diffraction optical tomography. Scientific reports 4, 6659 (2014). Yoon, J. et al. Label-free characterization of white blood cells by measuring 3D refractive index maps. Biomed. Opt. Express 6, 3865-3875 (2015). Myelodysplastic Syndrome by Three-dimensional Refractive Index Tomography. Laboratory Medicine Online 9, 185-188 (2019). phase microscopy and Zernike polynomials. Frontiers in Physics 7, 111 (2019). Moon, I., Anand, A., Cruz, M. \& Javidi, B. Identification of Malaria-infected red
and statistical inference. IEEE Photonics Journal 5, 6900207-6900207 (2013). algorithms with quantitative phase images of unstained cells. PloS one 11, e0163045 (2016). associated with early neurotoxic effects in Parkinson's disease. Cytometry part A 91, 510-518 (2017). Jourdain, P. et al. Determination of transmembrane water fluxes in neurons elicited by glutamate ionotropic receptors and by the cotransporters KCC2 and NKCC1: a digital holographic microscopy study. Journal of Neuroscience 31, 11846-11854 (2011). 
Cytometry Part A 91, 470-481 (2017).

Villone, M. M. et al. Full-angle tomographic phase microscopy of flowing quasi-spherical cells. Lab Chip 18, 126-131 (2018). Kim, T.-K. et al. Mitotic Chromosomes in Live Cells Characterized Using High-Speed and Label-Free Optical Diffraction Tomography. Cells 8, 1368 (2019). Oh, S. et al. In situ measurement of absolute concentrations by Normalized Raman Imaging. BioRxiv, 629543 (2019). Jiang, H. et al. Reconstruction of bovine spermatozoa substances distribution and morphological differences between Holstein and Korean native cattle using three-dimensional refractive index tomography. Scientific Reports 9, 8774 (2019). Cho, C. et al. Study of Optical Configurations for Multiple Enhancement of Microalgal Biomass Production. Scientific reports 9, 1723 (2019). Qin, Z. et al. Quantitative comparison of photothermal heat generation between gold nanospheres and nanorods. Scientific reports 6, 29836 (2016). Oh, J. et al. Optical Measurements of Three-Dimensional Microscopic Temperature Distributions Around Gold Nanorods Excited by Localized Surface Plasmon Resonance. Physical Review Applied 11, 044079 (2019). Lee, K., Kim, K., Kim, G., Shin, S. \& Park, Y. Time-multiplexed structured illumination using a DMD for optical diffraction tomography. Opt. Lett. 42, 999-1002, doi:10.1364/OL.42.000999 (2017). Shin, S., Kim, K., Yoon, J. \& Park, Y. Active illumination using a digital micromirror device for quantitative phase imaging. Opt. Lett. 40, 5407-5410, doi:10.1364/OL.40.005407 (2015). Shin, S. et al. in Quantitative Phase Imaging II. 971814 (International Society for Optics and Photonics). Wolf, E. Three-dimensional structure determination of semi-transparent objects from holographic data. Optics Communications 1, 153-156 (1969). Takeda, M., Ina, H. \& Kobayashi, S. Fourie
interferometry. JosA 72, 156-160 (1982). Debnath, S. K. \& Park, Y. Real-time quantitative phase imaging with a spatial phase-shifting algorithm. Opt. Lett. 36, 46774679 (2011). Gerchberg, R. Super-resolution through error energy reduction. Optica Acta: International Journal of Optics 21, 709-720 (1974). Lim, J. et al. Comparative study of iterative reconstruction algorithms for missing cone problems in optical diffraction tomography. Opt. Express 23, 16933-16948, doi:10.1364/OE.23.016933 (2015). the projection of maximal spatial bandwidths. JOSA A 35, 1891-1898 (2018). Kim, K. et al. High-resolution three-dimensional imaging of red blood cells parasitized by Plasmodium falciparum and in situ hemozoin crystals using optical diffraction tomography. Journal of biomedical optics 19, 011005 (2013). Lauer, V. New approach to optical diffraction tomography yielding a vector equation of diffraction tomography and a novel tomographic microscope. Journal of Microscopy 205, 165-176 (2002). Simon, B. \& Haeberlé, O. in Label-Free Super-Resolution Microscopy Nikoobakht, B. \& El-Sayed, M. A. Preparation and growth mechanism of gold nanorods (NRs) using seed-mediated growth method. Chemistry of Materials 15, 1957-1962 (2003). concentrations in intact red blood cells. Opt. Lett. 34, 3668-3670 (2009). Sung, Y. et al. Optical diffraction tomography for high resolution live cell imaging. Opt. Express 17, 266-277 (2009). Yoo, S., Kim, R., Park, J.-H. \&
ACS nano 10, 4274-4281 (2016). Popescu, G. et al. Optical imaging of cell mass and growth dynamics. American Journal of Physiology-Cell Physiology 295, C538-C544 (2008).

250-1100 nm dependent on concentration. Applied optics 45, 2838-2842 (2006).

61 Benga, G. et al. Effects of temperature on water diffusion in human erythrocytes and ghosts-nuclear magnetic resonance studies. Biochimica et Biophysica Acta (BBA)-Biomembranes 905, 339-348 (1987).

62 Yong, J. et al. Gold-Nanorod-Assisted Near-Infrared Stimulation of Primary Auditory Neurons. Advanced healthcare materials 3, 1862-1868 (2014).

63 Yoo, S., Hong, S., Choi, Y., Park, J.-H. \& Nam, Y. Photothermal inhibition of neural activity with near-infrared-sensitive nanotransducers. ACS nano 8, 8040-8049 (2014). Paviolo, C. et al. Laser exposure of gold nanorods can increase neuronal cell outgrowth. Biotechnology and bioengineering 110, 2277-2291 (2013).

65 Rastinehad, A. R. et al. Gold nanoshell-localized photothermal ablation of prostate tumors in a clinical pilot device study. Proceedings of the National Academy of Sciences 116, 18590-18596 (2019). 
bioRxiv preprint doi: https://doi.org/10.1101/2019.12.11.872218; this version posted December 12, 2019. The copyright holder for this preprint (which was not certified by peer review) is the author/funder, who has granted bioRxiv a license to display the preprint in perpetuity. It is made available under aCC-BY 4.0 International license.

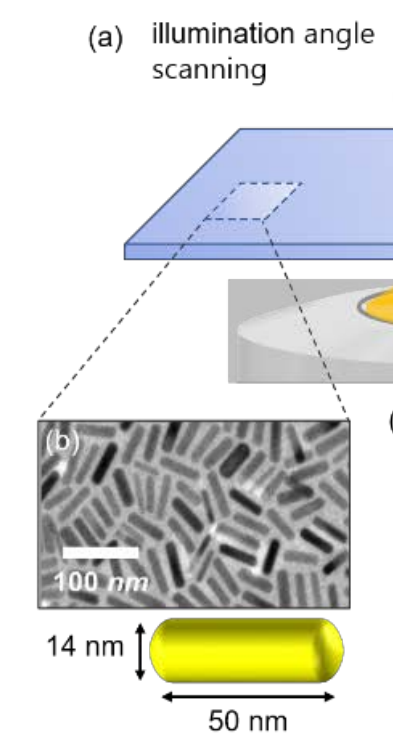

(e)
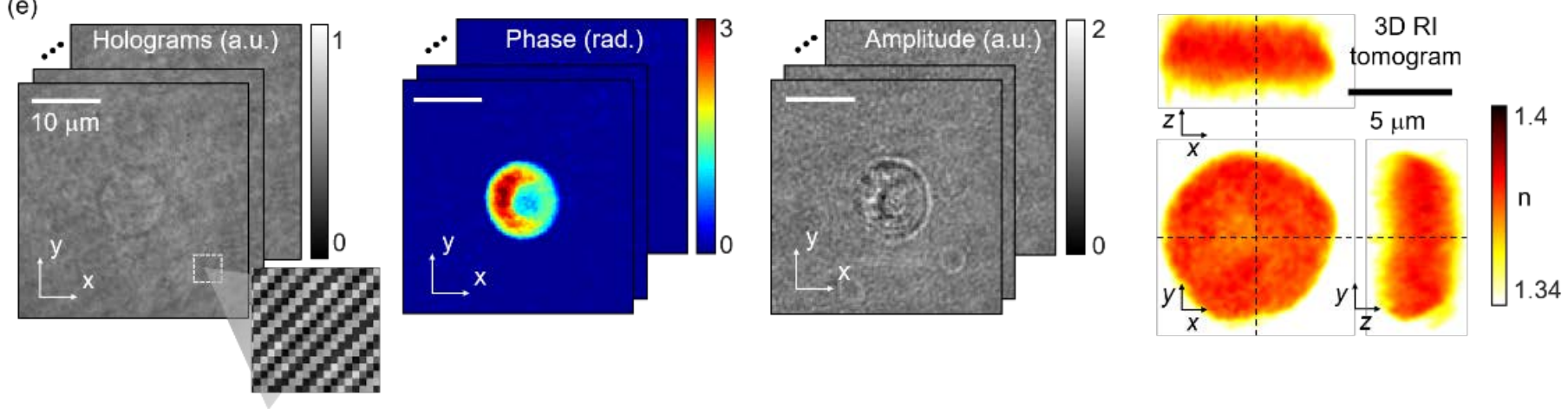

Fig. 1 (a) Experimental schematics. RBC samples were heated by GNRs excited by NIR illumination. (b) Transmission electron microscopy of GNRs and the GNR shape. (c) Measured absorption spectrum of the GNR coated coverslip ${ }^{40}$. (d) Optical setup. The 3-D morphology of individual RBCs is measured using ODT equipped with a DMD, while the sample is excited by the NIR laser. (e) ODT reconstruction process. The 3-D RI tomogram is reconstructed from the measured multiple 2D holograms of a sample. 
bioRxiv preprint doi: https://doi.org/10.1101/2019.12.11.872218; this version posted December 12, 2019. The copyright holder for this preprint (which was not certified by peer review) is the author/funder, who has granted bioRxiv a license to display the preprint in perpetuity. It is made available under aCC-BY 4.0 International license.

(a)

No NIR excitation (Original RBC)

(c)

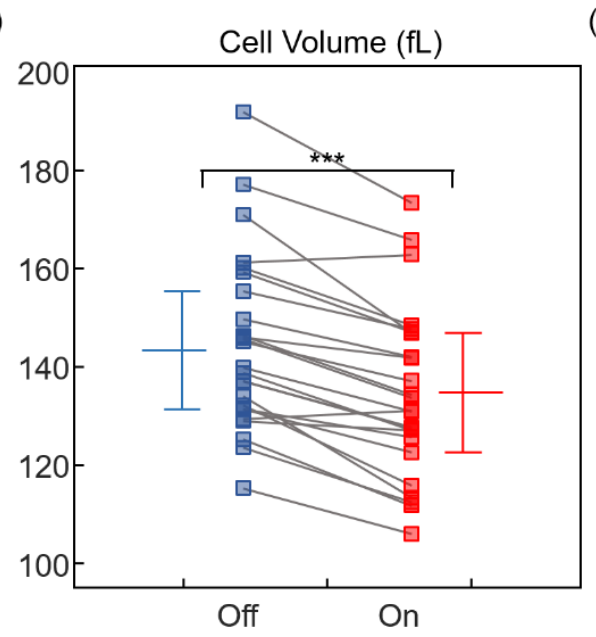

(d)

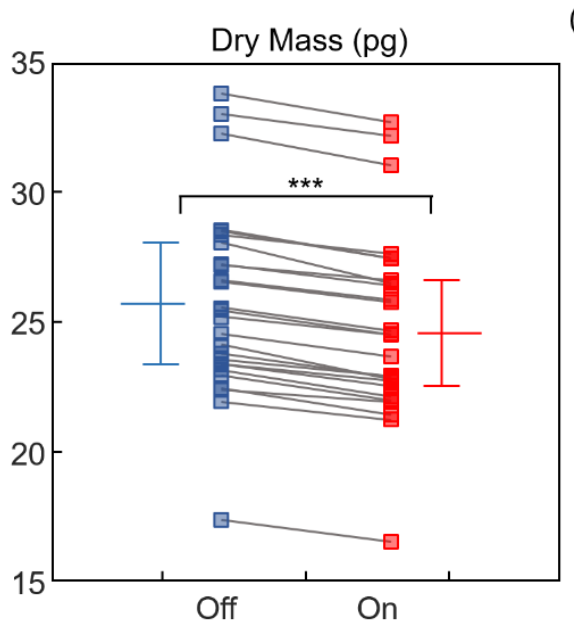

NIR excitation (power $=0.72 \mathrm{~mW}$ )

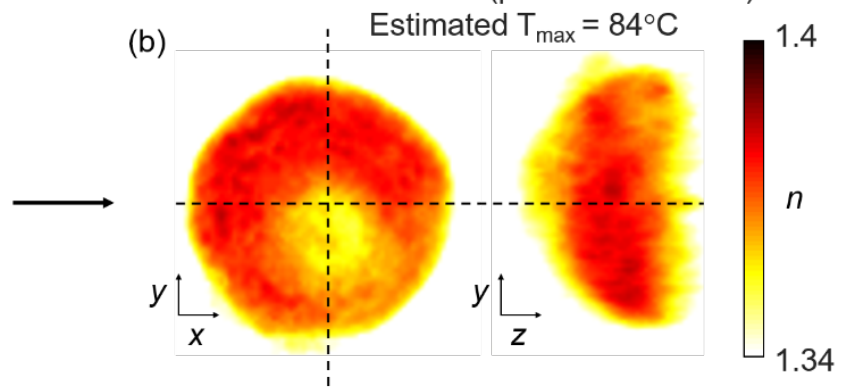

(e)

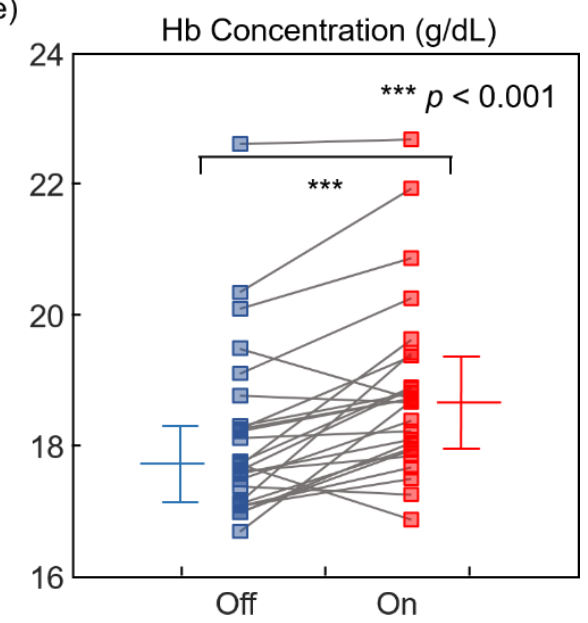

Fig. 2 (a,b) Reconstructed 3-D RI tomograms of representative RBCs before and after NIR excitation. (c) Cell volume, (d) dry mass, (e) Hb concentration for all measured RBCs before and after NIR excitation exposure with a power of 0.72mW $(n=33)$.

Each square denotes an individual RBC measurement. The horizontal lines indicate the mean values, and error bars represent standard deviation. 
bioRxiv preprint doi: https://doi.org/10.1101/2019.12.11.872218; this version posted December 12, 2019. The copyright holder for this preprint (which was not certified by peer review) is the author/funder, who has granted bioRxiv a license to display the preprint in perpetuity. It is made available under aCC-BY 4.0 International license.
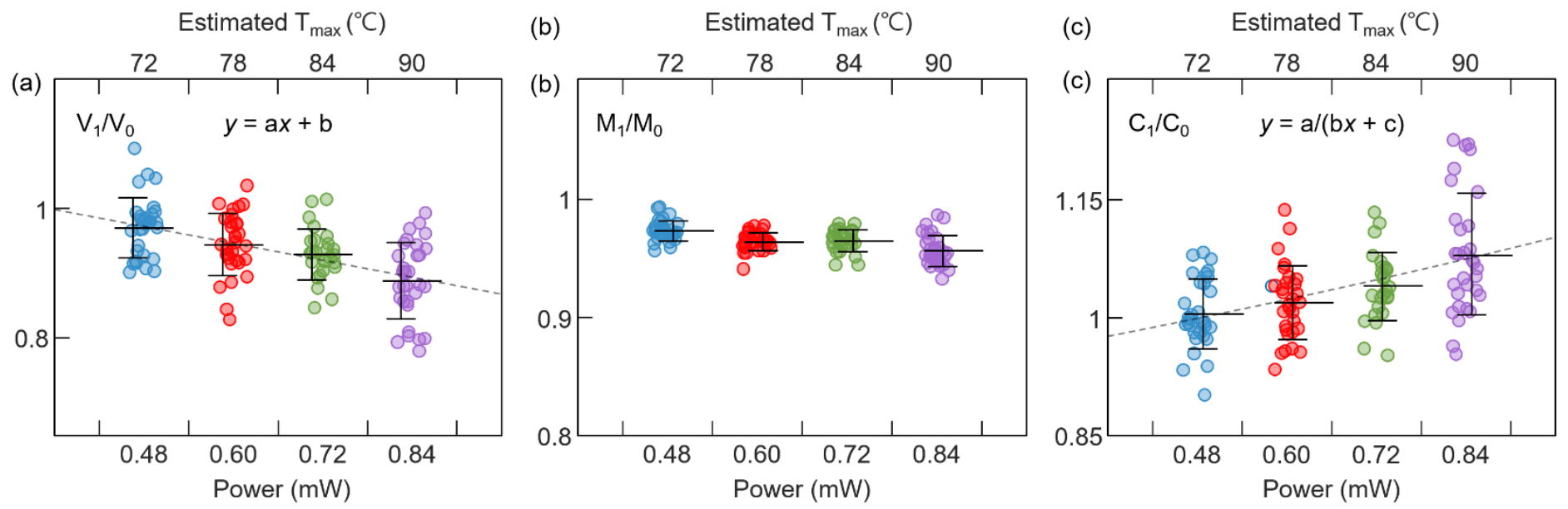

Fig. 3 (a) Relative RBC volume before and after heat treatment $\left(\mathrm{V}_{1} / \mathrm{V}_{0}\right)$ for each $\mathrm{RBC}$ with respect to NIR excitation power. Gray dotted line is linearly fitted graph that describe relationship between average value of $\mathrm{V}_{1} / \mathrm{V}_{0}$ and the excitation power. Coefficients are statistically defined as $\mathrm{a}=-21.7833\left[\mathrm{~mW}^{-1}\right]$ and $\mathrm{b}=1.286$ with $\mathrm{p}$-values of 0.0167 and $1.30 \times 10^{-3}$ for each parameter, respectively. (b) Relative RBC dry mass before and after heat treatment $\left(\mathrm{M}_{1} / \mathrm{M}_{0}\right)$ for each RBC with respect to NIR excitation power. (c) Relative RBC Hb concentration before and after heat treatment $\left(\mathrm{C}_{1} / \mathrm{C}_{0}\right)$ for each RBC with respect to the NIR excitation power. Gray dotted line is rationally fitted graph that describe relationship betwwen average value of $C_{1} / C_{0}$ and the excitation power. Coefficients are statistically defined as a $=0.0154\left[\mathrm{~mW}^{-1}\right], \mathrm{b}=-0.30209$ and $\mathrm{c}=82.453$ with p-values of $5.70 \times 10^{-4}$, 0.0164 , and $2.11 \times 10^{-4}$, respectively. Each circle denotes an individual RBC measurement; $n=[33,30,33,33]$ for $[0.48,0.60$, 0.72, 0.84] mW NIR power respectively. The center horizontal line indicates the mean values, and the error bar indicates the standard deviation. 
bioRxiv preprint doi: https://doi.org/10.1101/2019.12.11.872218; this version posted December 12, 2019. The copyright holder for this preprint (which was not certified by peer review) is the author/funder, who has granted bioRxiv a license to display the preprint in perpetuity. It is made available under aCC-BY 4.0 International license.
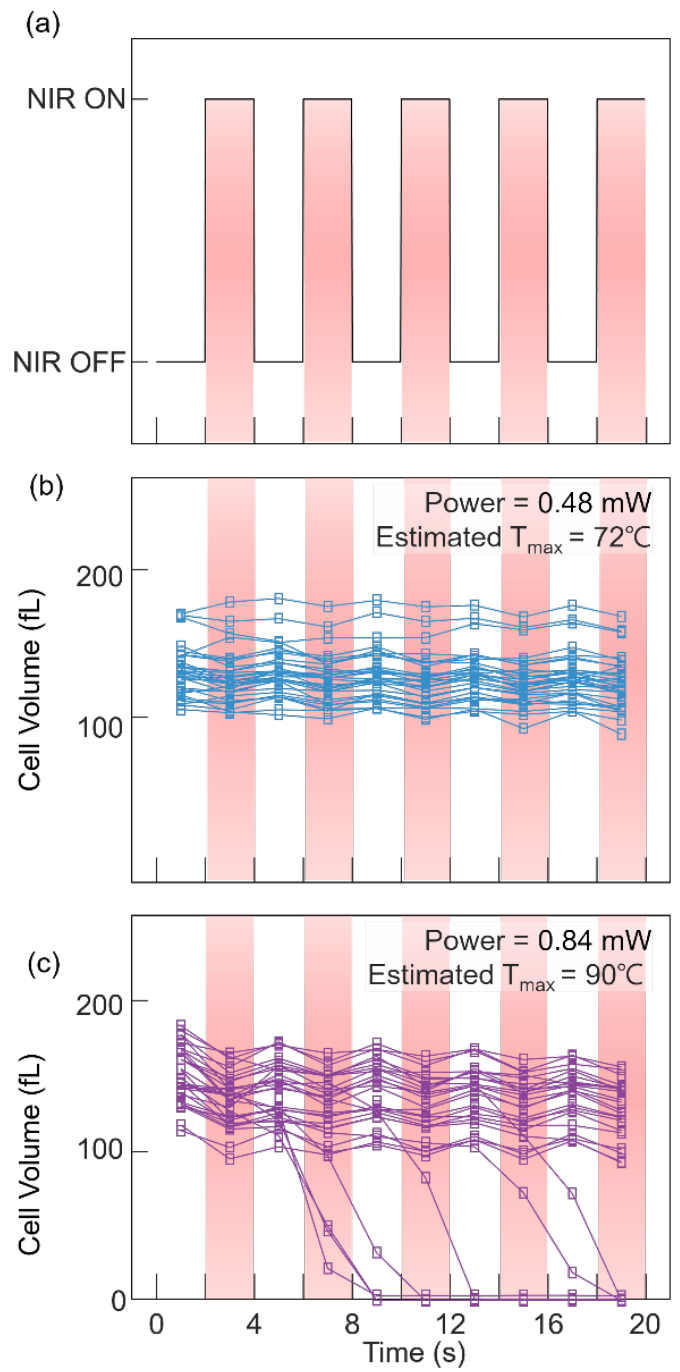

(d) Power $=0.48 \mathrm{~mW}$ Estimated $\mathrm{T}_{\max }=72^{\circ} \mathrm{C}$
Power $=0.60 \mathrm{~mW}$ Estimated $\mathrm{T}_{\max }=78^{\circ} \mathrm{C}$
Power $=0.72 \mathrm{~mW}$ Estimated $\mathrm{T}_{\max }=84^{\circ} \mathrm{C}$
Power $=0.84 \mathrm{~mW}$ Estimated $\mathrm{T}_{\max }=90^{\circ} \mathrm{C}$
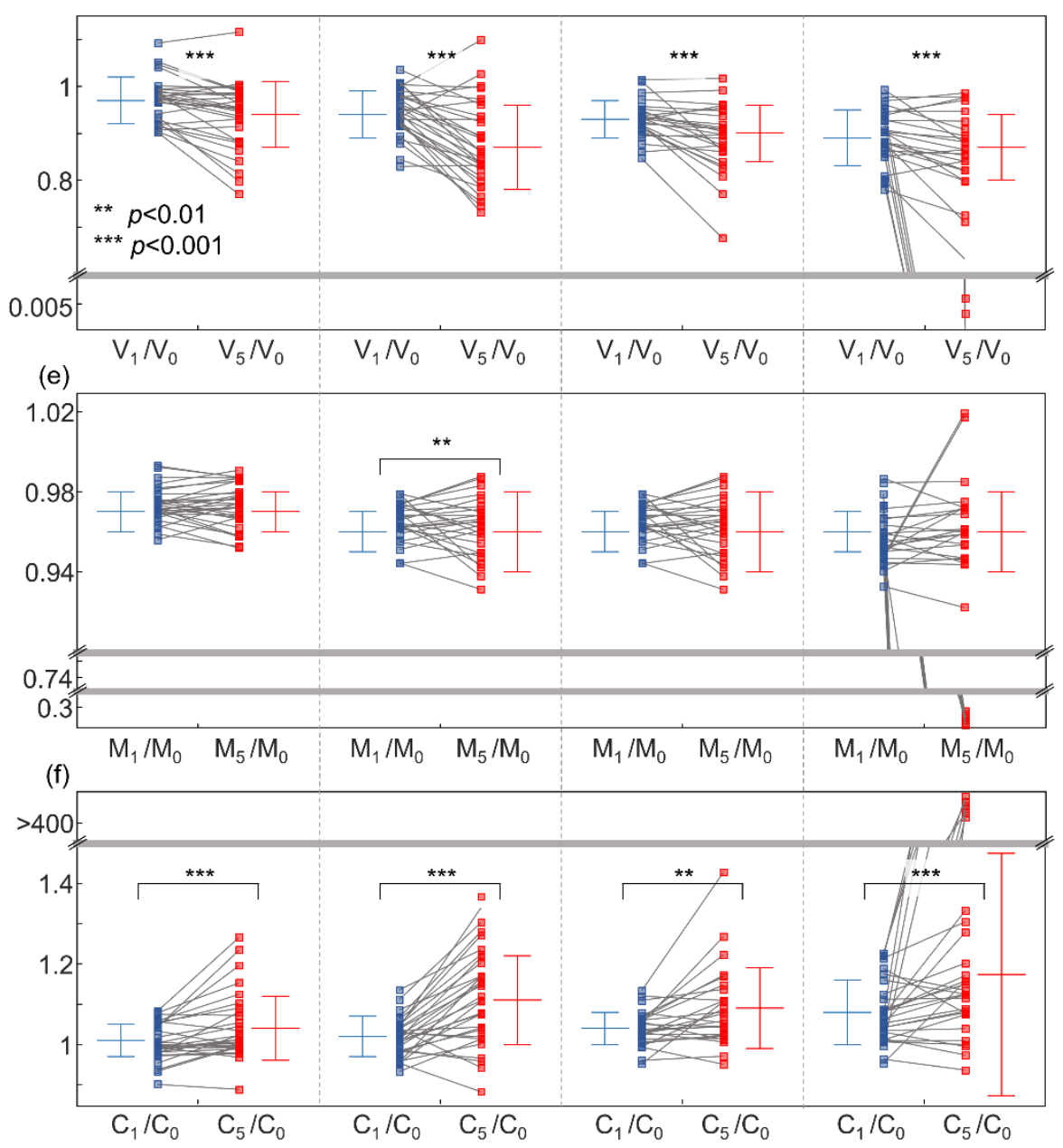

Fig. 4 (a) NIR excitation power profile with respect to time is presented. Individually traced plot of cell volume of RBCs with NIR power of (b) $0.48 \mathrm{~mW}$ and (c) $0.84 \mathrm{~mW}$. Calculated (d) cell volume $\left(\mathrm{V}_{i} / \mathrm{V}_{0}\right)$, (e) dry mass $\left(\mathrm{M}_{i} / \mathrm{M}_{0}\right)$, and (f) protein concentration $\left(\mathrm{C}_{i} / \mathrm{C}_{0}\right)$, where $i$ indicates the $i$ th step of heat exposure. Each blue and red square denotes an individual RBC measurement for the first heat exposure step and the fifth heat exposure step. The center horizontal line indicates the mean values, and the error bar indicates the standard deviation; $n=[33,30,33,33]$ for $[0.48,0.60,0.72,0.84] \mathrm{mW}$ of NIR power respectively. ** and $* * *$ denote that the $p$-value is smaller than 0.01 and 0.001 , respectively. 